Research Paper

\title{
Repair of Critical-Sized Mandible Defects in Aged Rat Using Hypoxia Preconditioned BMSCs with Up-regulation of Hif-1 $\alpha$
}

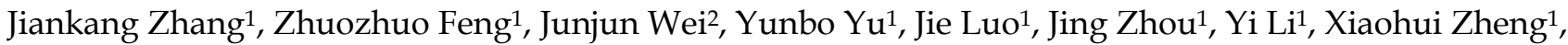
Wei Tang1, Lei Liu'1 ${ }^{1}$ Jie Long1, Xiaoyu Li1 ${ }^{1}$, Wei Jing ${ }^{1 凶}$

1. State Key Laboratory of Oral Diseases, National Clinical Research Center for Oral Diseases, Department of Oral and Maxillofacial Surgery, West China Hospital of Stomatology, Sichuan University, Chengdu 610041, China

2. Department of Stomatology, the Second Affiliated Hospital of Zhengzhou University, Zhengzhou 450014, China

$\triangle$ Corresponding author: Wei Jing, MD, State Key Laboratory of Oral Diseases, National Clinical Research Center for Oral Diseases, Department of Oral and Maxillofacial Surgery, West China Hospital of Stomatology, Sichuan University, Chengdu 610041, China. Tel: 86-028-85503406; E-mail: jingwei@scu.edu.cn.

(C) Ivyspring International Publisher. This is an open access article distributed under the terms of the Creative Commons Attribution (CC BY-NC) license (https://creativecommons.org/licenses/by-nc/4.0/). See http://ivyspring.com/terms for full terms and conditions.

Received: 2017.12.03; Accepted: 2018.02.20; Published: 2018.03.11

\begin{abstract}
The repair of bone defects in the geriatric population remains a challenge for modern medicine. Transplantation of bone marrow mesenchymal stem cells (BMSCs) combined with or without biomaterials has been a promising approach to bone restoration and regeneration. Typically, the transplanted BMSCs are cultured under normoxic conditions $\left(21 \% \mathrm{O}_{2}\right.$ and $10 \%$ serum medium) in vitro. However, the micro-environment of bone defect area is much more severe, in which lower physiological oxygen tension $(<1 \%)$ and tissue ischemia were present. Therefore, how to improve the survival rate and osteogenesis of transplanted BMSCs at the low oxygenic and ischemic region in vivo is critical. Hypoxia inducible factor-la (HIF-la) plays an important role in the tolerance, angiogenesis and osteogenesis of BMSCs during bone regeneration after transplantation. Previous studies have demonstrated that Dimethyloxaloylglycine (DMOG) improves the angiogenic activity of BMSCs. Typically, angiogenesis and osteogenesis are coupled with each other. Therefore, we detected that hypoxia preconditioned BMSCs with the combined treatment of $1 \% \mathrm{O}_{2}$ and $0.5 \mathrm{mM}$ DMOG showing up-regulation of Hif- $1 \alpha$ could enhance the survival rate of BMSCs under severe condition (serum-free medium and $1 \% \mathrm{O}_{2}$ ) in vitro and enhances the angiogenesis and osteogenesis potential of BMSCs under $1 \% \mathrm{O}_{2}$ microenvironment in vitro. The hypoxia preconditioned BMSCs were transplanted into critical-sized mandible defects in aged SD rats to test the effectiveness of hypoxic preconditioning approach. We found that hypoxia preconditioned BMSCs improved the repair of critical-sized mandible defects in vivo. These data showed that hypoxia preconditioned BMSCs with the up-regulation of Hif- l $\alpha$ have the potential of enhancing the bone healing process in geriatric individuals.
\end{abstract}

Key words: aging; BMSCs; hypoxia inducible factor-1a; mandible.

\section{Introduction}

The regeneration of bone defects caused by severe trauma or tumor resection remains a challenge for modern medicine. In the geriatric population, aging leads to reduced bone intensity and BMSCs in aged individuals present a trend of decreased osteogenic differentiation and increased adipogenic differentiation which directly leads to the decrease in bone repairing ability [1, 2]. BMSCs from aged individuals have reduced wound healing, angiogenesis, proliferation and anti-apoptotic capabilities [3]. The transplantation of BMSCs has become a promising approach for bone regeneration in the aged population. However, poor cell survival rate in vivo after implantation has been one of the 
major limitations of the usefulness of this therapy, most of the implanted cells being lost within the first few days after transplantation to the hypoxia and ischemic environment [4]. Previous studies reported that HIF-1a enable cells to survive in oxygen deprivation condition by providing oxygen-independent adenosine triphosphate (ATP) production or by inhibiting apoptosis induced by hypoxia [5]. However, HIF-1a protein, but not HIF-1 $\alpha$ gene,is ubiquitylated and degraded by the $26 S$ proteasome rapidly under normoxic condition [6, 7]. And the poor survival rate may be caused by the degredation of HIF-1a protein. Therefore, up-regulating the protein expression level of HIF-1a may enhance the survival rate of the transplanted BMSCs in the severe environment of bone defect area. And then enhance the angiogensis and osteogenesis capacity.

BMSCs are multipotent stem cells capable of proliferating in vitro under conditions in which they retain undifferentiated and can differentiate into osteoblasts, chondrocytes, and adipocytes with appropriate induction $[8,9]$. BMSCs play a critical role in the process of fracture healing and bone regeneration. Typically, in regenerative medicine and tissue engineering, BMSCs are cultured under ambient or normoxic conditions $\left(21 \% \mathrm{O}_{2}\right)$ in vitro for research before transplanted into vivo, while BMSCs resides under hypoxic oxygen tensions $\left(1 \% \mathrm{O}_{2}\right)$ in vivo [10]. The endogenic defenses of BMSCs against oxidative stress may be destroyed by free radicals generated in these culture conditions, leading to the decreased utility of these cells [11]. The oxygen tensions of bone defects area are even lower than $1 \%$ owing to the atypical blood vessel networks $[12,13]$. When the BMSCs are used as seed cell to regenerate bone defects, the BMSCs cultured in normoxic condition must adapt from $21 \% \mathrm{O}_{2}$ and $10 \%$ serummedium in culture to $<1 \% \mathrm{O}_{2}$ and ischemia in bone defect area. However most of the implanted cells are lost within the first few days after transplantation to the low oxygen tension and ischemic tissue. Here we try to demonstrate that hypoxia preconditioned BMSCs with $0.5 \mathrm{mM}$ DMOG and $1 \% \mathrm{O}_{2}$ enhanced the survival rate in low oxygen tension and ischemic environment and improved angiogenesis and osteogenesis potential in vitro and in vivo.

\section{Materials and Methods}

\section{Bone marrow-derived mesenchymal stem cells isolation and culture}

Cells and tissues from animals were obtained according to governing ethical principles, and this study was approved by the Institutional Review
Board (IRB) of the West China Hospital of Stomatology. Total BMSCs were isolated from 4-week-old male SD rats (Sichuan University Animal Experimental Center, Chengdu, China). Cells were cultured as previously described [14, 15]. Briefly, BMSCs were isolated from the whole bone marrow (tibia and femur) of 4-week-old male SD rats, both ends of the tibia and femur were cut off at the epiphysis, and the marrow was flushed out using a-MEM (GIBCO BRL, Grand Island, NY, USA) supplemented with $10 \%$ fetal bovine serum (FBS; Invitrogen, Carlsbad, CA, USA), 100 units $/ \mathrm{mL}$ penicillin and $100 \mathrm{mg} / \mathrm{mL}$ streptomycin. Cells were cultured at a density of $4 \times 10^{4} / \mathrm{cm}^{2}$ in flasks in a-MEM at $37^{\circ} \mathrm{C}$ in moist air and $5 \% \mathrm{CO}_{2}$. After $48 \mathrm{~h}$ of the initial culture, BMSCs adhered to the bottom of the plastic culture flasks whereas other non-adherent cells were discarded by change medium. The medium is replaced every $48 \mathrm{~h}$. Cells were passaged at a ratio of 1:3 when expanded to $90 \%$ confluence. Cells were used for the experiments after three passages.

\section{Proliferation capacity and Colony-Forming Unit-Fibroblast (CFU-F) Assay}

The proliferation capacity of BMSCs was measured with Cell Counting Kit-8 (CCK-8, Dojindo, WST, Japan) after being cultured in hypoxic or normoxic condition. Cells at passage 3 were seeded in quintuplicate at a density of 2500 cells/well in 96-well plates and incubated in hypoxic and normoxic condition respectively. One plate was tested at each time point (days 1-4) using a CCK-8 according to the manufacturer's protocol. Briefly, at each time point, $100 \mu \mathrm{l}$ a-MEM containing and $10 \mu \mathrm{l} \mathrm{CCK-8}$ was added to each well and incubated at $37^{\circ} \mathrm{C}$ for $2 \mathrm{~h}$, and then the absorbance was measured at $450 \mathrm{~nm}$.

CFU-F assays were performed as described [16-18]. One hundred passage 3 BMSCs were planted on $10-\mathrm{cm}$ tissue culture dishes in triplicate in growth medium and cultured under hypoxic and normoxic condition respectively with medium changes every 3 days. After 14 days, CFU-F cultures were washed with phosphate-buffered saline (PBS), fixed with $4 \%$ paraformaldehyde, and then stained with crystal violet dye. Washing the colonies with PBS again and removing residual dye. Differences in colonies number were evaluated by a two-tailed one-sample $t$ test to block for variability between groups.

\section{Survival rate assays under $1 \% \mathrm{O}_{2}$ in serum-free medium}

Survival rate of BMSCs after they were engrafted in bone defect area was evaluated. The combination of $1 \% \mathrm{O}_{2}$ and serum-free medium was intended to mimic the micro environment of bone defect [19]. Live \& 
Dead Viability Assay Kit (KeyGEN Biotech, Nanjing, China) was applied to assess the number of surviving cells. The BMSCs at passage 3 were seeded in triplicate at a density of $2 \times 10^{5}$ cells/well in 6-well plates and incubated under $1 \% \mathrm{O}_{2}$ and serum-free medium condition. And the cells in control groups were incubated under $21 \% \quad \mathrm{O}_{2}$ and $10 \%$ serum medium condition. Live \& Dead Viability staining was taken according to the protocol at the time points of $24 \mathrm{~h}$ and $48 \mathrm{~h}$. Images were taken in three randomly chosen fields per well, and all image data were analyzed and quantified by Image Pro Plus 6.0 software to calculate the total number of survived cells. Differences in number of surviving cells were evaluated by a two-tailed one-sample $t$ test to block for variability between groups.

\section{Survival rate of BMSCs cultured under $1 \% \mathrm{O}_{2}$ in serum-free medium with or without hypoxic precondition}

BMSCs were cultured to passage 3 under normoxic conditions. There are 3 steps for hypoxia preconditioning. Firstly, passage 3 BMSCs were exposed to fresh complete medium supplemented with $0.5 \mathrm{mM}$ prolyl hydroxylase inhibitor, DMOG under $21 \% \mathrm{O}_{2}$ condition for 48 hours. Secondly, passage 3 BMSCs were exposed to fresh medium under $1 \% \mathrm{O}_{2}$ condition for $48 \mathrm{~h}$. Finally, passage 3 BMSCs were exposed to fresh complete medium supplemented with $0.5 \mathrm{mM}$ DMOG under $1 \% \mathrm{O}_{2}$ condition for 48 hours. $1 \% \mathrm{O}_{2}$ condition was achieved with a trigas incubator containing $5 \% \mathrm{CO}_{2}, 1 \% \mathrm{O}_{2}$, and $94 \% \mathrm{~N}_{2}$, and the $\mathrm{O}_{2}$ concentration was maintained using pure $\mathrm{N}_{2}$. And then hypoxia preconditioned BMSCs were incubated under $1 \% \mathrm{O}_{2}$ in a serum-free medium. After 48 hours, Live \& Dead Viability Assay Kit was applied to assess the number of surviving cells. Images were taken in three randomly chosen fields per well, and all image data were analyzed and quantified by Image Pro Plus 6.0 software to calculate the total number of survived cells. Differences in the number of surviving cells were evaluated by a two-tailed one-sample $t$ test to block for variability between groups.

\section{Tube formation test}

Tube formation on Matrigel (BD Biosciences, San Jose, CA) is a well-established assay to detect the formation of three-dimensional vessels and assess angiogenesis capacity in vitro. Tube formation test was performed as described [20]. Matrigel was prepared in 48-well plate according to the protocol. BMSCs were preconditioned with or without DMOG in $21 \% \quad \mathrm{O}_{2}$ and $1 \% \mathrm{O}_{2}$ for $48 \mathrm{~h}$. And then preconditioned BMSCs were seeded into Matrigel and incubated at $37^{\circ} \mathrm{C}, 21 \% \mathrm{O}_{2}$ and $5 \% \mathrm{CO}_{2}$. Each culture well was photographed $6 \mathrm{~h}$ later. The total length of capillary-like structures was quantified in five randomly chosen fields using Image Pro Plus 6.0 software. Differences in tubes length were evaluated by a two-tailed one-sample $t$ test to block for variability between groups.

\section{Western blot analysis}

Western blotting was taken to analyze the protein expression of HIF-1a and vascular endothelial growth factor (VEGF). Total protein was harvested from cells in different groups. Cells were washed twice with PBS and lysed with modified RIPA buffer (50 mM HEPES, $\mathrm{pH} 7.3,1 \%$ sodium deoxycholate, 1 $\mathrm{mM}$ ethylenediamine tetraacetic acid, $150 \mathrm{mM} \mathrm{NaCl}$, $1 \%$ Triton X-100, 0.1\% SDS $1 \mathrm{mM}, 1 \mathrm{mM} \mathrm{NaF}$, and protease inhibitor cocktail) on ice for $30 \mathrm{~min}$ and centrifuged at $14,000 \times \mathrm{g}$ at $4^{\circ} \mathrm{C}$ for $30 \mathrm{~min}$. Protein concentrations were measured using the BCA protein assay kit (KeyGEN Biotech, Nanjing, China). Equal amounts of protein extracts were fractionated on $8 \%$ SDS-polyacrylamide gels and transferred to nitrocellulose membrane $(0.22 \mu \mathrm{m}$, Millipore, Bedford, MA, USA). Membranes were incubated with specific primary antibodies of HIF-1a (Cell Signalling Technology, Catalog number 14179) and VEGF (ab1316, 1:1000; Abcam) at 1:1000 overnight at $4^{\circ} \mathrm{C}$. $\beta$-actin was used as the control. Washing the membrane with Tris-buffered saline with Tween (TBST; 20mM Tris-HCl [pH 7.6], 1\% Tween 20, $137 \mathrm{mM} \mathrm{NaCl}$ ), then probed with the indicated primary antibodies, and detected immunoreactive products using ECL Plus Western blotting detection reagent, and photographed by Quantity One software (Bio-Rad, CA, USA).

\section{ELISA assay and qRT-PCR analysis}

The secretion amount of VEGF was measured in triplicate with an ELISA kit (Bioswap, Wuhan, China) according to the protocol. And for the qRT-PCR analysis, total RNA was isolated using TRIzol (Invitrogen, CA, USA) from hypoxia preconditioned BMSCs and regular BMSCs, followed by reverse transcription with the PrimeScript RT Reagent Kit (TaKaRa, Shiga, Japan). The real-time polymerase chain reaction (RT-PCR) was performed using SYBR Premix Ex Taq (Takara) according to the manufacturer's instruction. $\beta$-actin was used as the control. The relative quantification of gene expression level was determined using the DDct method.

\section{Osteogenic differentiation}

The differentiation potential of BMSCs with or without hypoxic precondition was analyzed with 
specific inductive media in $1 \% \mathrm{O}_{2}$ condition. Cells were seeded in a 6 -well plate at a density of $2 \times 10^{5}$ cells per well, and then incubated in osteogenic inductive medium (a-MEM, 10\% FBS, $100 \mathrm{U} / \mathrm{mL}$ penicillin and $100 \mathrm{mg} / \mathrm{mL}$ streptomycin, $0.1 \mathrm{mM}$ dexamethasone, 10 $\mathrm{mM} \beta$-glycerophosphate, and $50 \mathrm{mM}$ vitamin $\mathrm{C})$. The medium was replaced every 3 days. Alkaline phosphatase (ALP) was assessed by ALP staining and semi-quantitative analysis of ALP was taken according to the protocol at day 7. Briefly, the Osteogenic induced BMSCs were lysed using RIPA lysis buffer (KeyGEN Biotech, Nanjing, China), and then the supernatant was collected, and then transfered $50 \mu \mathrm{ll}$ into a 96-well plate. Then chromogenic substrate and detection buffer from Alkaline Phosphatase Assay Kit (Beyotime) were added, then incubated for $10 \mathrm{~min}$ at $37{ }^{\circ} \mathrm{C}$. The ALP activity was tested at the $405 \mathrm{~nm}$ wave length. Calcium deposition was assessed by Alizarin Red S (ARS) staining after 3 weeks induction.

\section{Animal Experiments}

The experiment was performed on the 21-month-old, male SD rats and the body weight was about 500-600g. After BMSCs were hypoxia preconditioned with $0.5 \mathrm{mM}$ DMOG under $1 \% \mathrm{O}_{2}$ condition, $1 \times 10^{5}$ cells were seeded on a gelatin sponge (GS) [21]. GS was used as negative control for critical size defects repair. All animals were anesthetized by intraperitoneal injection of pentobarbital. A $1-\mathrm{cm}$ incision overlying and paralleling with the right mandible was made. The inferior border of the mandible was exposed by blunt dissection. A critical-sized defect was created by means of a $5-\mathrm{mm}$ diameter trephine burr (Fig. 6A) [22, 23]. And then GS, GS with regular BMSCs, and GS with hypoxia preconditioned BMSCs (Fig. 6B, C) were placed in the defects respectively. The incision was closed with non-resorbable suture. Mandibles were harvested 8 and 12 weeks after implantation.

\section{Micro-CT Measurement}

All animals were sacrificed and right mandibles were harvested for Micro-CT analysis at 8 weeks after implantation. Volume analysis of new bone formation was performed including bone volume (BV) and bone volume/total volume (BV/TV).

\section{Histological evaluation and Immunohistochemistry}

Mandibles were harvested 12 weeks after implantation, and fixed in a $10 \%$ neutral buffered formalin solution. The fixed specimens were decalcified under $10 \%$ ethylenediaminetetraacetic acid (EDTA) solution under shaking for 8 weeks. Decalcified specimens were embedded in paraffin and cut into sections at a thickness of $5 \mathrm{um}$. The sections were stained with hematoxylin and eosin (H\&E) and Masson's trichrome solution, respectively. Immunohistochemistry for osteocalcin (OCN, ab13420, 1:100; Abcam), Runx2 (ab23981, 1:100; Abcam), VEGF (ab1316, 1:100; Abcam) and CD31 (ab24590, 1:200; Abcam) were then performed in the sections from each group.

\section{Statistical analysis}

Quantified data are expressed as mean values SD. We used SPSS software (version 19.0) to compute statistical significance with Student's paired t-test. $\mathrm{P}<0.05$ was considered statistically significant. Experiments were performed in triplicate.

\section{Results}

\section{Hypoxia had effects on the proliferation potential of BMSCs.}

To explore the effects of hypoxia on cell proliferation capacity, we performed proliferation assays using CCK-8 kit and CFU-F assay in $1 \% \mathrm{O}_{2}$ and $21 \% \mathrm{O}_{2}$ groups. The proliferative capacity of BMSCs cultured under $1 \% \mathrm{O}_{2}$ decreased to a minimum in $48 \mathrm{~h}$ and then increased, whereas BMSCs cultured under $21 \% \mathrm{O}_{2}$ was increased gradually. And at day 4 , the cell number of BMSCs cultured under $1 \% \mathrm{O}_{2}$ exceed that under $21 \% \mathrm{O}_{2}$ condition (Fig. 1A). There was a significant difference in absorbance index between $1 \%$ $\mathrm{O}_{2}$ and $21 \% \mathrm{O}_{2}$ groups at the time point of $48 \mathrm{~h}$ (Fig. 1B). BMSCs colonies were observed at each oxygen tension after 14 days in culture. Colony formation was significantly improved under $1 \% \mathrm{O}_{2}$ compared with that in $21 \% \mathrm{O}_{2}$ group. The difference in the number of colonies was observed at $21 \% \mathrm{O}_{2}$ and $1 \% \mathrm{O}_{2}$ (Fig. 1C, D).

\section{The survival rates of BMSCs decreased seriously in severe condition in vitro.}

Exposure to hypoxia and a serum-free medium condition had negative effects on cell survival rate. After $24 \mathrm{~h}$, the number of surviving cells was $1.7 \times 10^{5}$ in $1 \% \mathrm{O}_{2}$ and serum-free medium condition. And the number of surviving cells was $2.5 \times 10^{5}$ in $21 \% \mathrm{O}_{2}$ and $10 \%$ serum-medium (Fig. $2 \mathrm{~A}, \mathrm{C}$ ). The survival rate was $67.8 \%$. After $48 \mathrm{~h}$, only $6.3 \times 10^{4}$ cells survived in $1 \% \mathrm{O}_{2}$ and serum-free medium condition. And there were $4.7 \times 10^{5}$ survived cells in $21 \% \quad \mathrm{O}_{2}$ and $10 \%$ serum-medium condition (Fig. 2B, C). And the survival rate was $13.23 \%$. This indicates that most of the implanted BMSCs are lost within the first 2 days after transplantation to the hypoxic and ischemic environment which representing the microenvironment of bone defect. 
A
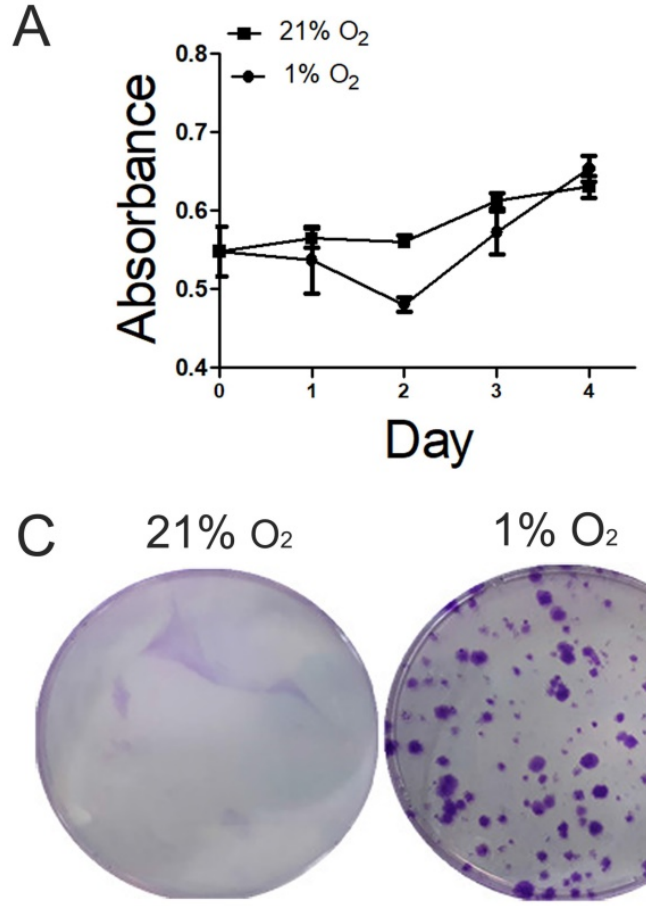

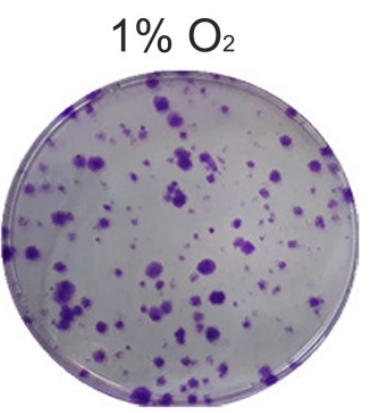

B
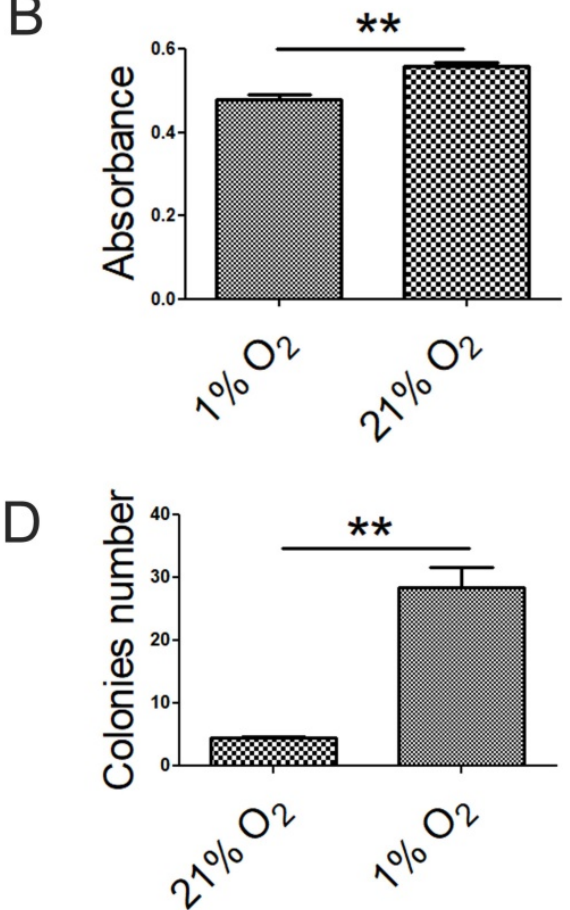

Figure 1. Effect of oxygen tensions on the proliferation potential of BMSCs. (A) The proliferative capacity of BMSCs cultured under $1 \% \mathrm{O}_{2}$ decreased to a minimum in 48h and then increased, whereas BMSCs cultured under $21 \% \mathrm{O}_{2}$ increased gradually. (B) There was significant difference in absorbance index between $1 \% \mathrm{O}_{2}$ group and $21 \% \mathrm{O}_{2}$ group at the time point of $48 \mathrm{~h}$. (C) For CFU assays, crystal violet staining showed that BMSCs increased in colony number when cultured under $1 \% \mathrm{O}_{2}$ condition for 14 days. (D) The number of colonies was counted. Colonies less than $2 \mathrm{~mm}$ in diameter and faintly stained colonies were ignored. *, p $<0.05$; $* *, p<$ 0.01 .

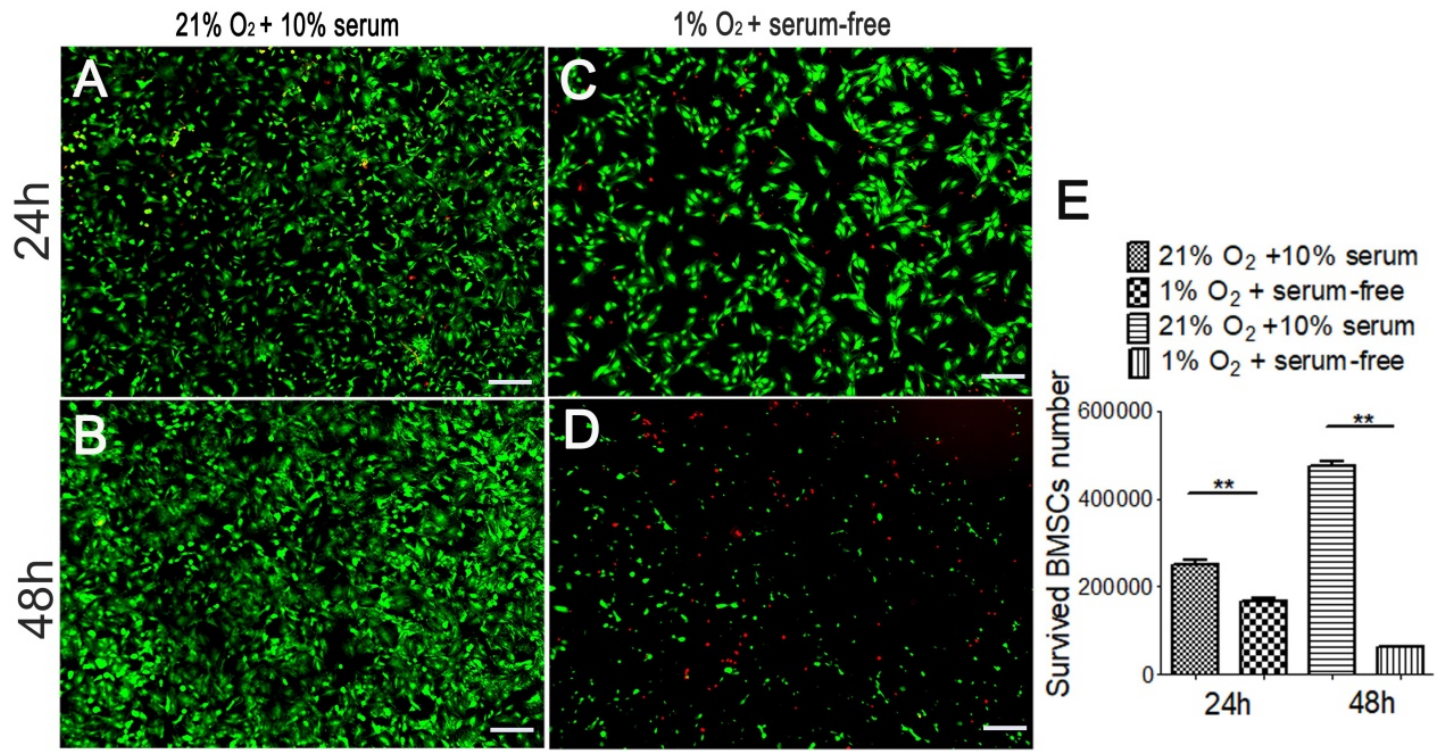

Figure 2. The number of survived BMSCs in severe condition. The number of survived BMSCs cultured in regular condition $\left(21 \% \mathrm{O}_{2}\right.$ and $10 \%$ serum medium) for $24 \mathrm{~h}(\mathrm{~A})$ and for $48 \mathrm{~h}(\mathrm{~B})$, as well as the number of survived BMSCs cultured in severe condition ( $1 \% \mathrm{O}_{2}$ and serum-free medium) for $24 \mathrm{~h}(\mathrm{C})$ and for $48 \mathrm{~h}(\mathrm{D})$ were detected respectively using Live/dead assay. (E) The number of survived BMSCs cultured in serum-free medium and $1 \% \mathrm{O}_{2}$ condition is lower than that of the BMSCs culture in the regular condition. **, $\mathrm{p}<0.01$; green represents live BMSCs, red represents dead BMSCs; scale bar $=50 \mu \mathrm{m}$.

\section{Hypoxia preconditioning improves the survived BMSCs number in severe condition in vitro}

Hypoxia preconditioning could increase the tolerance of BMSCs, and the survived BMSCs number was examined in $1 \% \quad \mathrm{O}_{2}$ and serum-free medium condition. Cells in different groups were culture in $21 \% \mathrm{O}_{2}$ and $1 \% \mathrm{O}_{2}$ respectively for 48 hours before being transferred into a severe condition (hypoxic and serum-free). After being transferred and cultured for $48 \mathrm{~h}$ in $1 \% \mathrm{O}_{2}$ and serum-free medium, the number of 
surviving BMSCs in hypoxia preconditioned group was more than that of in the control group without hypoxic preconditioning (Fig.3A-D). The number of surviving BMSCs cultured in normoxic condition was $8.3 \times 10^{3}$. The number of surviving cells number was $3.9 \times 10^{4}$ in the DMOG-treated group. The number of surviving cells in the $1 \% \mathrm{O}_{2}$ treated group was $1.2 \times 10^{5}$. The number of surviving cells in DMOG combined with $1 \% \mathrm{O}_{2}$ treated group was $2.2 \times 10^{5}$ (Fig. $3 \mathrm{E})$. The protein of HIF-1a was at a higher expression level in hypoxia preconditioned BMSCs (Fig. 3F). This indicates that hypoxia preconditioned BMSCs have an increased capacity of survival in hypoxia and ischemic environment, and HIF-1a may participates in the process.

\section{Hypoxia preconditioning enhanced angiogenic activities of BMSCs in vitro}

The effect of hypoxia preconditioning on BMSC-induced angiogenesis was examined by tube formation stimulated by Matrigel in vitro. Hypoxia preconditioned BMSCs formed more tubes (Fig. 4A) and the total tube length was significantly longer than that formed by normally cultured BMSCs. The total tube length of DMOG preconditioned BMSCs was 1.90 folds compared with that of normally cultured BMSCs, the total tube length of $1 \% \mathrm{O}_{2}$ preconditioned BMSCs was 2.64 folds compared with that of normally cultured BMSCs, and the $0.5 \mathrm{mM}$ DMOG with $1 \% \mathrm{O}_{2}$ preconditioned BMSCs was 3.27 folds compared with that of normally cultured BMSCs (Fig. 4B). The
mRNA expression level of VEGF examined by qRT-PCR was higher in hypoxia preconditioned groups than that of in the regular groups. And the expression level in $0.5 \mathrm{mM}$ combined with $1 \% \mathrm{O}_{2}$ preconditioned group was the highest (Fig. 4C). The expression of angiogenic proteins in different groups was detected using western blot analysis and ELISA kit. The significantly higher expression level of VEGF, the downstream factors of HIF-1a, was seen in hypoxia preconditioned BMSCs compared with that of regular BMSCs (Fig. 4D, E). These data support the hypothesis that angiogenic inductive effects in BMSCs can be enhanced by hypoxia preconditioning.

\section{Hypoxia preconditioning enhanced osteogenetic activities of BMSCs in vitro}

On day 7 and 21 after osteogenic induction, ALP activity was significantly enhanced in the hypoxia preconditioned groups (Fig. 5A). Semi-quantitative analysis of ALP activity was taken, and the supernatant absorbance index under $405 \mathrm{~nm}$ was higher in hypoxia preconditioned BMSCs than that in regular BMSCs (Fig. 5B). ALP activity in hypoxia preconditioned groups was higher than that in a regular group on day 7. Furthermore, ARS staining shows that more mineralization nodules were formed in hypoxia preconditioned BMSCs after induced cultured for 21 days (Fig. 5C). All of these results show that hypoxia preconditioned BMSCs with up-regulation of HIF-1a promotes osteogenic activity.

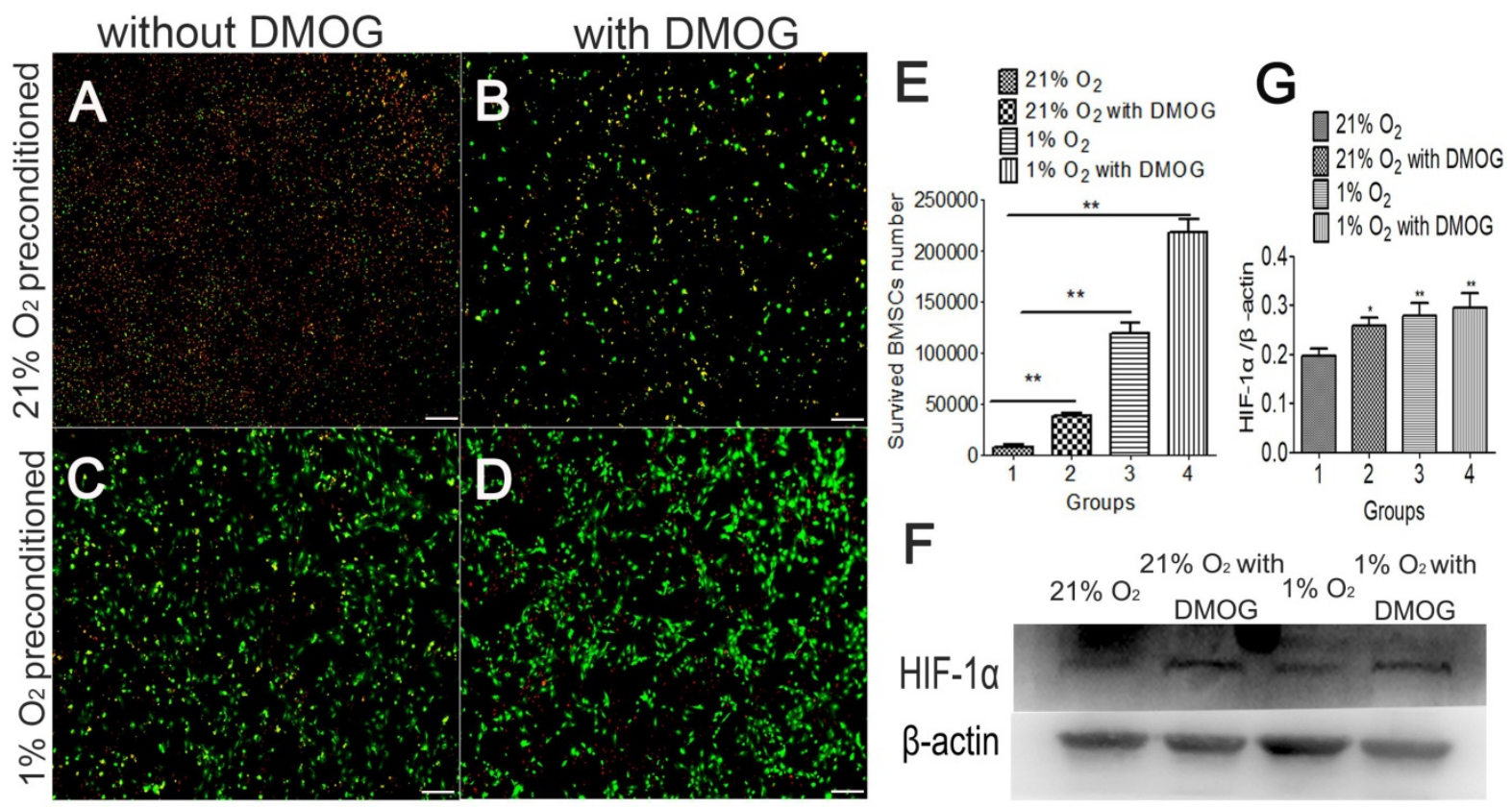

Figure 3. Hypoxia preconditioning improves the tolerance and survival rate of $\mathrm{BMSCs}$ after they were transferred into $1 \% \mathrm{O}_{2}$ and serum-free conditions. (A) $21 \%$ $\mathrm{O}_{2}$ preconditioned BMSCs without DMOG, (B) $21 \% \mathrm{O}_{2}$ preconditioned BMSCs with 0.5mM DMOG, (C) $1 \% \mathrm{O}_{2}$ preconditioned BMSCs without DMOG, and (D) $1 \%$ $\mathrm{O}_{2}$ preconditioned BMSCs with 0.5mM DMOG. (E) The survived cell number of hypoxia preconditioned BMSCs is higher than that of BMSCs cultured in the regular condition after transferred into serum-free medium and $1 \% \mathrm{O}_{2}$ condition for $48 \mathrm{~h}$ abruptly. (F) Western blot shows hypoxia preconditioning for $48 \mathrm{~h}$ enhanced HIF-1 $\alpha$ expression level, $\beta$-actin was used as the Internal control. **, $p<0.01$; green represents live BMSCs, red represents dead BMSCs, scale bar=50 $\mu$ m. 
A

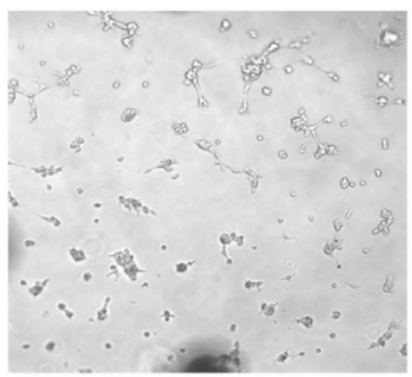

B

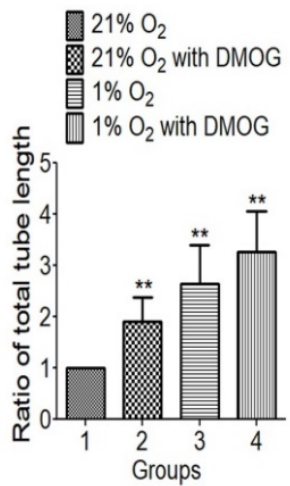

$21 \% \mathrm{O}_{2}$ with DMOG
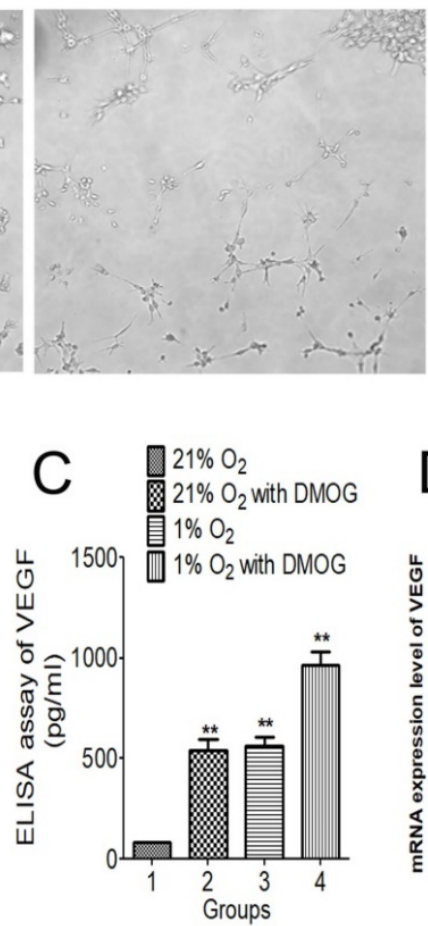

$1 \% \mathrm{O}_{2}$

$1 \% \mathrm{O}_{2}$ with DMOG

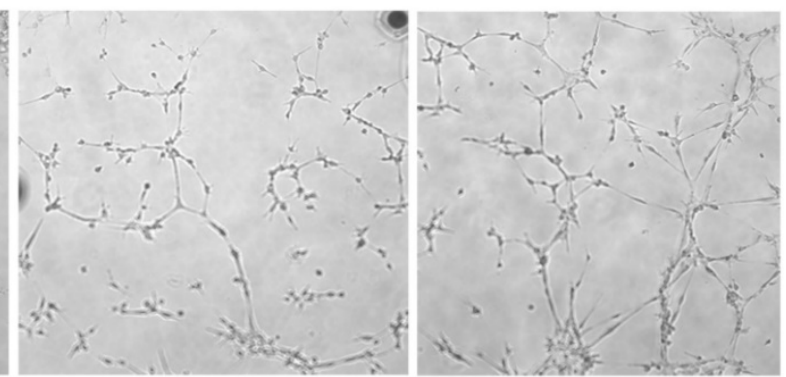

Figure 4. Hypoxia preconditioning enhanced angiogenic activities of $\mathrm{BMSCs}$ in $21 \% \mathrm{O}_{2}$ and $10 \%$ serum medium condition. (A) $21 \%$ O $\mathrm{O}_{2}$ preconditioned $\mathrm{BMSCs}$ without DMOG for $48 \mathrm{~h}, 21 \% \mathrm{O}_{2}$ preconditioned BMSCs with $0.5 \mathrm{mM}$ DMOG for $48 \mathrm{~h}, 1 \% \mathrm{O}_{2}$ preconditioned BMSCs without DMOG for $48 \mathrm{~h}$, and $1 \% \mathrm{O}_{2}$ preconditioned BMSCs with $0.5 \mathrm{mM} \mathrm{DMOG}$ for $48 \mathrm{~h}$ and then were transferred into Matrigel and incubated at $37^{\circ} \mathrm{C}, 21 \% \mathrm{O}_{2}$ and $5 \% \mathrm{CO}$ for $6 \mathrm{~h}$. (B) The fold change of tube length showed hypoxia preconditioned $\mathrm{BMSCs}$ formed more tubes than that of $\mathrm{BMSCs}$ cultured in regular condition in $21 \% \mathrm{O}_{2}$ and $10 \%$ serum medium condition. (C) The secretion of VEGF in hypoxia preconditioned BMSCs was at significantly higher level than that of in regular BMSCs. (D) The mRNA levels of VEGF in hypoxia preconditioned BMSCs for $48 \mathrm{~h}$ was significantly increased compared to that in regular BMSCs. (E) Western blot showed hypoxia preconditioning for 48 $\mathrm{h}$ enhanced VEGF protein expression level of BMSSCs, $\beta$-actin was used as the Internal control. **, $p<0.01$.

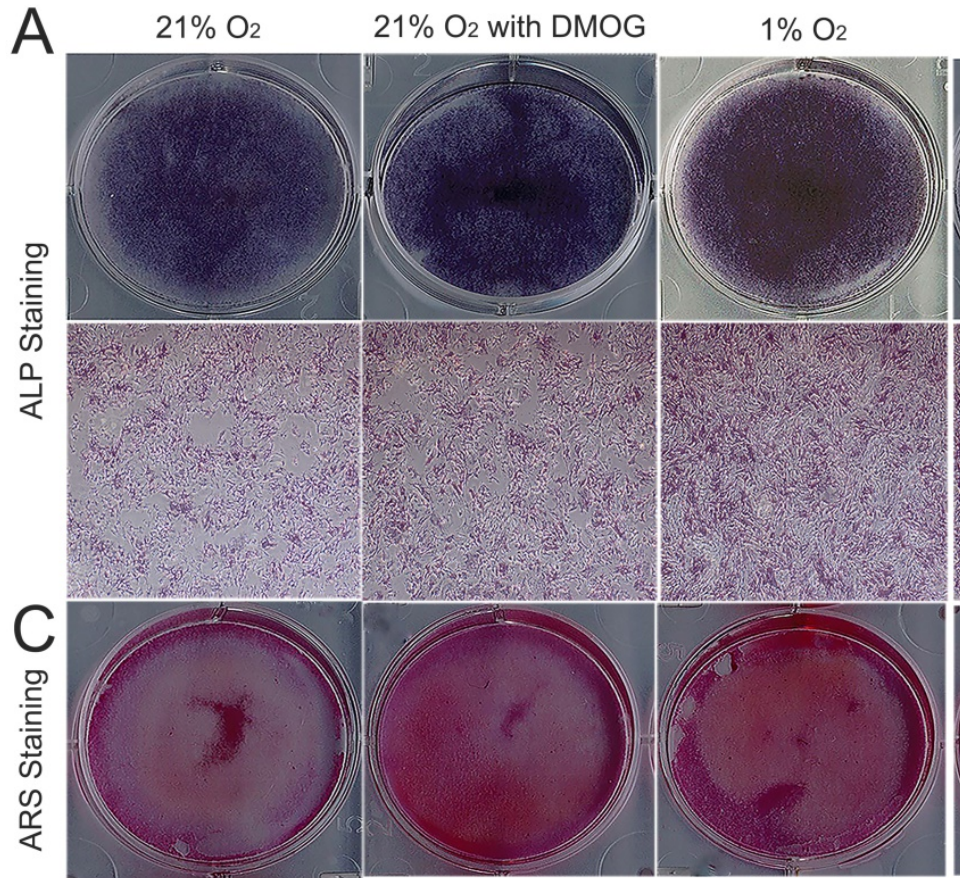

$1 \% \mathrm{O}_{2}$ with DMOG

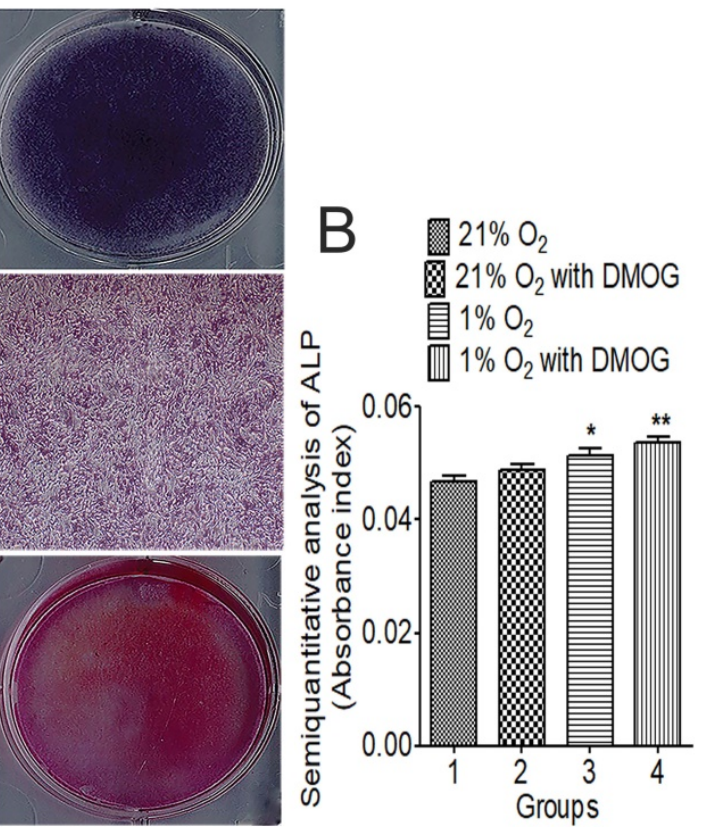

Figure 5. Hypoxia preconditioning enhanced osteogenesis activities of $\mathrm{BMSCs}$ in vitro. $21 \% \mathrm{O}_{2}$ preconditioned $\mathrm{BMSCs}$ without $\mathrm{DMOG}$ for $48 \mathrm{~h}, 21 \% \mathrm{O}_{2}$ preconditioned BMSCs with $0.5 \mathrm{mM} \mathrm{DMOG}$ for $48 \mathrm{~h}, 1 \% \mathrm{O}_{2}$ preconditioned $\mathrm{BMSCs}$ without DMOG for $48 \mathrm{~h}$, and $1 \% \mathrm{O}_{2}$ preconditioned BMSCs with $0.5 \mathrm{mM}$ DMOG for $48 \mathrm{~h}$ were then transferred into osteogenic differentiation medium and incubated at $37^{\circ} \mathrm{C}, 1 \% \mathrm{O}_{2}$ and $5 \% \mathrm{CO}_{2}$ for 21 days. (A) ALP staining and (B) semiquantitative analysis of ALP activity were taken at day 7. The supernatant absorbance index under $405 \mathrm{~nm}$ was higher in hypoxia preconditioned BMSCs than that in regular BMSCs. (C) Alizarin red S staining shows that more mineralization nodules were formed in hypoxia preconditioned BMSCs after induced cultured for 21 days. *, $><0.05 ; * *, p<0.01$. 


\section{Hypoxia preconditioning improved the healing of critical-sized mandible defects of aged rats in vivo}

To evaluate newly formed bones in an aged rat model, micro-CT examination was performed 8 weeks after the implantation of BMSCs in defect areas. The newly formed plate-like bone structure was visible in the bone defect area 8 weeks after treatment with target preconditioned BMSCs. The newly formed bone in $1 \% \mathrm{O}_{2}$ with DMOG preconditioned BSMCs group expanded to most of the bone defect area (Fig. $6 \mathrm{D})$. The ratio of new $\mathrm{BV}$ to total $\mathrm{BV}(\mathrm{BV} / \mathrm{TV}, \%)$, which indicates the relative amount of newly formed bone, was $20.25 \%$ in the GS group, $22.53 \%$ in the GS+BMSCs group, and $36.87 \%$ in the GS+hypoxia preconditioned BMSCs group (Fig. 6E, F). Quantification of newly formed bone showed the hypoxia preconditioned BMSCs group had up to 1.25-fold more newly formed bone volume compared with that in the regular BMSCs group at 8 weeks. These results illustrate that hypoxia preconditioning is able to increase bone repairing capacity of BMSCs in vivo when transplanted into aged bony defects.
Hypoxia preconditioned BMSCs formed more newly bone and vessels in the bone defect area of aged rats in vivo

The newly formed tissue was microscopically evaluated by H\&E and Mallory's trichrome staining 12 weeks after transplantation. In groups of GS, most of the newly formed tissues were muscular tissue, while the group of GS+BMSCs only showed a little of fibrous tissue. And more fibrous tissues and newly formed bone was seen in the GS+hypoxia preconditioned BMSCs group compared with that in the first two groups (Fig. 7). The Immunohistochemistry for VEGF and CD31 showed there were more newly formed vessels in the group of GS+hypoxia preconditioned BSCs, and nearly no newly formed vessels in the group of GS (Fig. 8A, B). Immunohistochemistry for Runx 2 and OCN showed osteogenetic activity was at a higher level in GS+hypoxia preconditioned BMSCs group compared with that in other groups (Fig. 8C, D). Meanwhile, the micro-CT and the H\&E staining results described previously were confirmed again by these immunohistochemistry results for Runx 2 and $\mathrm{OCN}$.

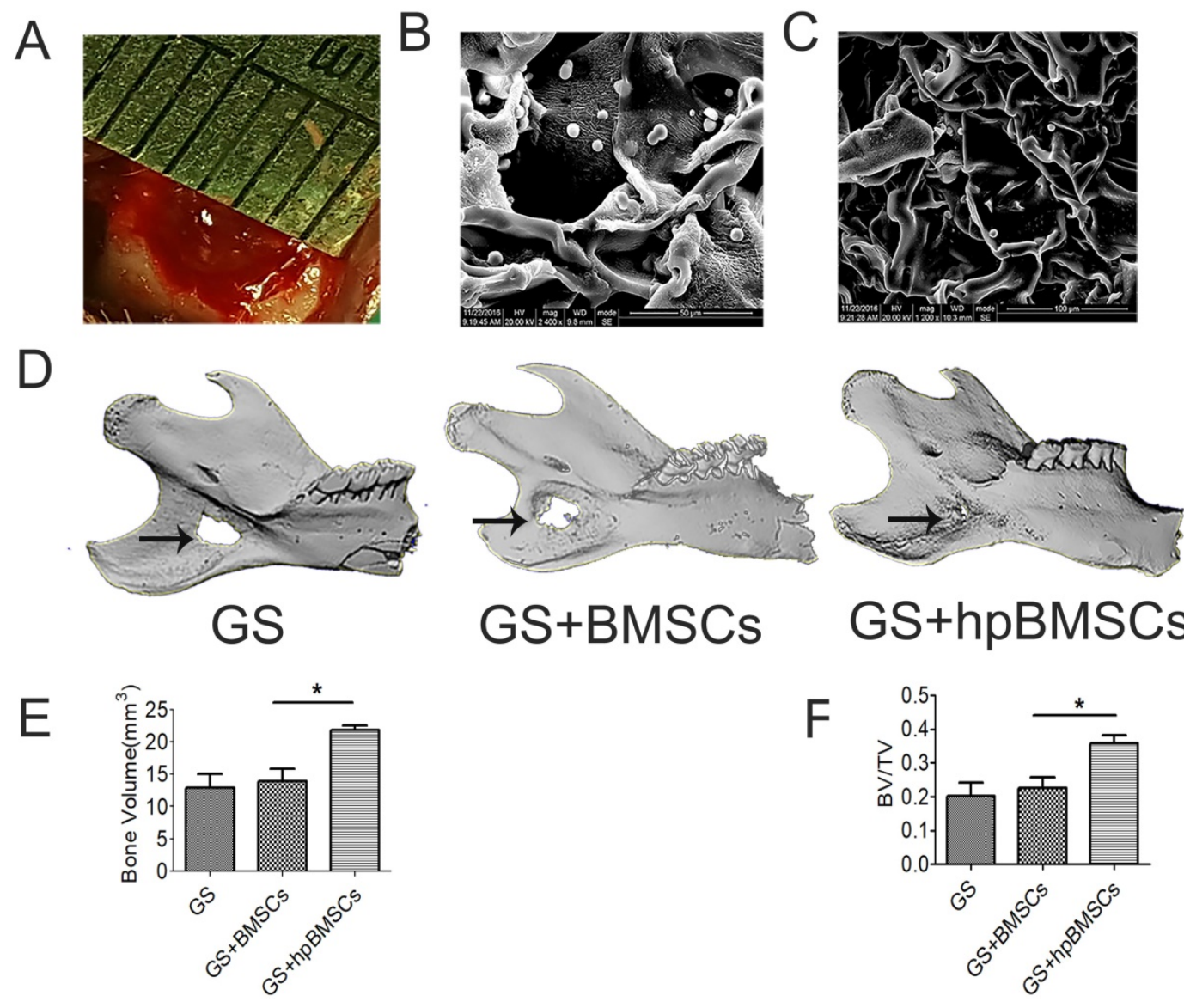

Figure 6. Osteogenesis in the bone defect area of aged rats was evaluated using Micro-CT at 8 weeks after implantation. (A) The critical-sized defects on the mandible of aged rats. $(B, C)$ the morphology of the GS combined with BMSCs using scanning electron microscope (SEM) analysis. (D) Micro-CT 3D reconstruction images showed that the new bone formation in GS+hpBMSCs was more than in other two groups. (E-F) Quantitative analysis of bone volume (BV) and bone volume/tissue volume (BV/TV) of the newly formed bone in the defects area showed that there was more newly formed bone in the GS+hpBMSCs group than that in other two groups. *, $\mathrm{p}<0.05 ; * *, \mathrm{p}<0.01$. GS, Gelatin Sponge. Arrow, bone defect area in mandible. hpBMSCs, hypoxia preconditioned BMSCs. 


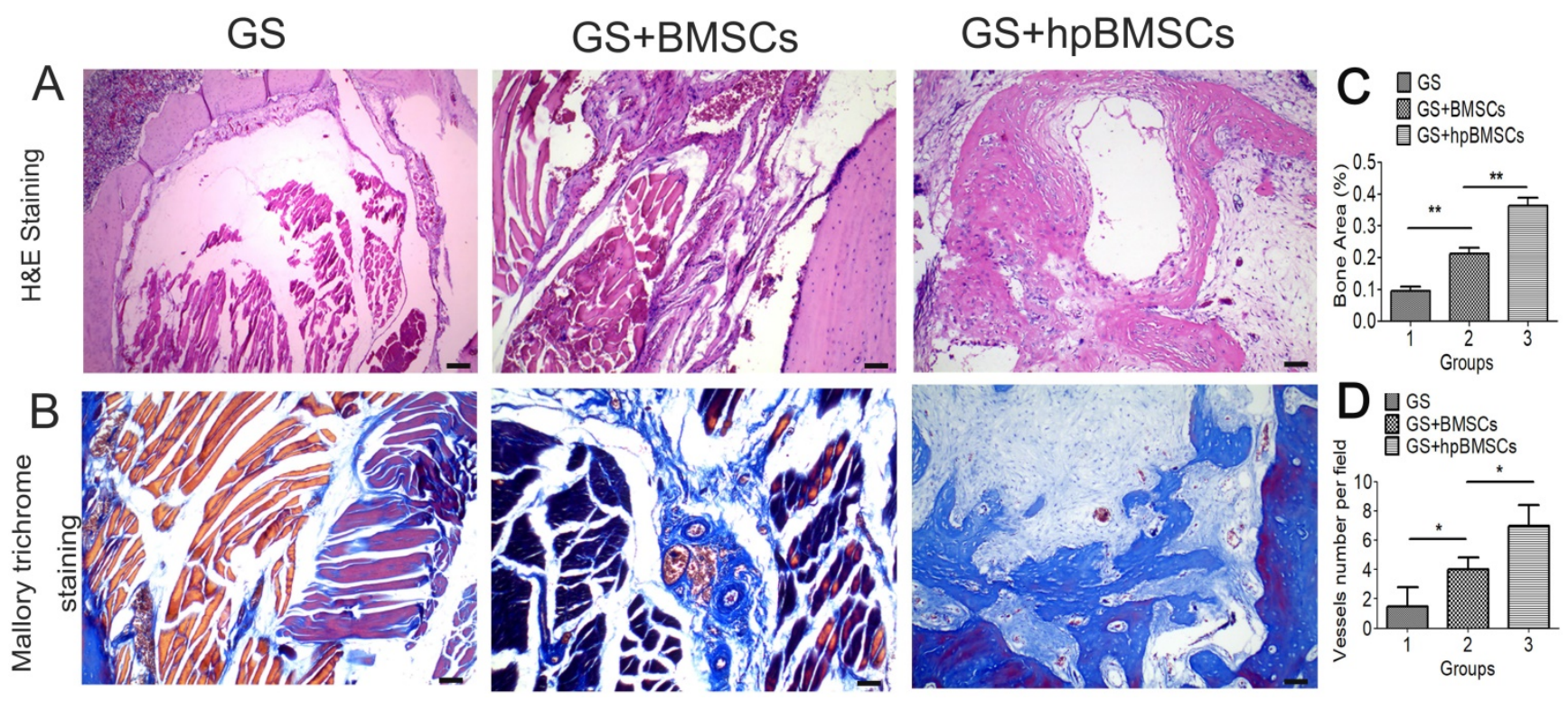

Figure 7. Bone regeneration capacity in the bone defect area of aged rats was evaluated by histological analysis at 12 weeks after implantation. (A) H\&E staining for morphologic evaluation and (B) Mallory trichrome staining for collagen in each group. Most of the newly formed tissue in GS group was muscular tissue. (C)There was more newly formed bone in the GS+hpBMSCs group than that in other two groups.(D) the quantification of newly formed vessels in CD31 staining. (Scale bars $=50 \mu \mathrm{m}$. hpBMSCs, hypoxia preconditioned BMSCs.)
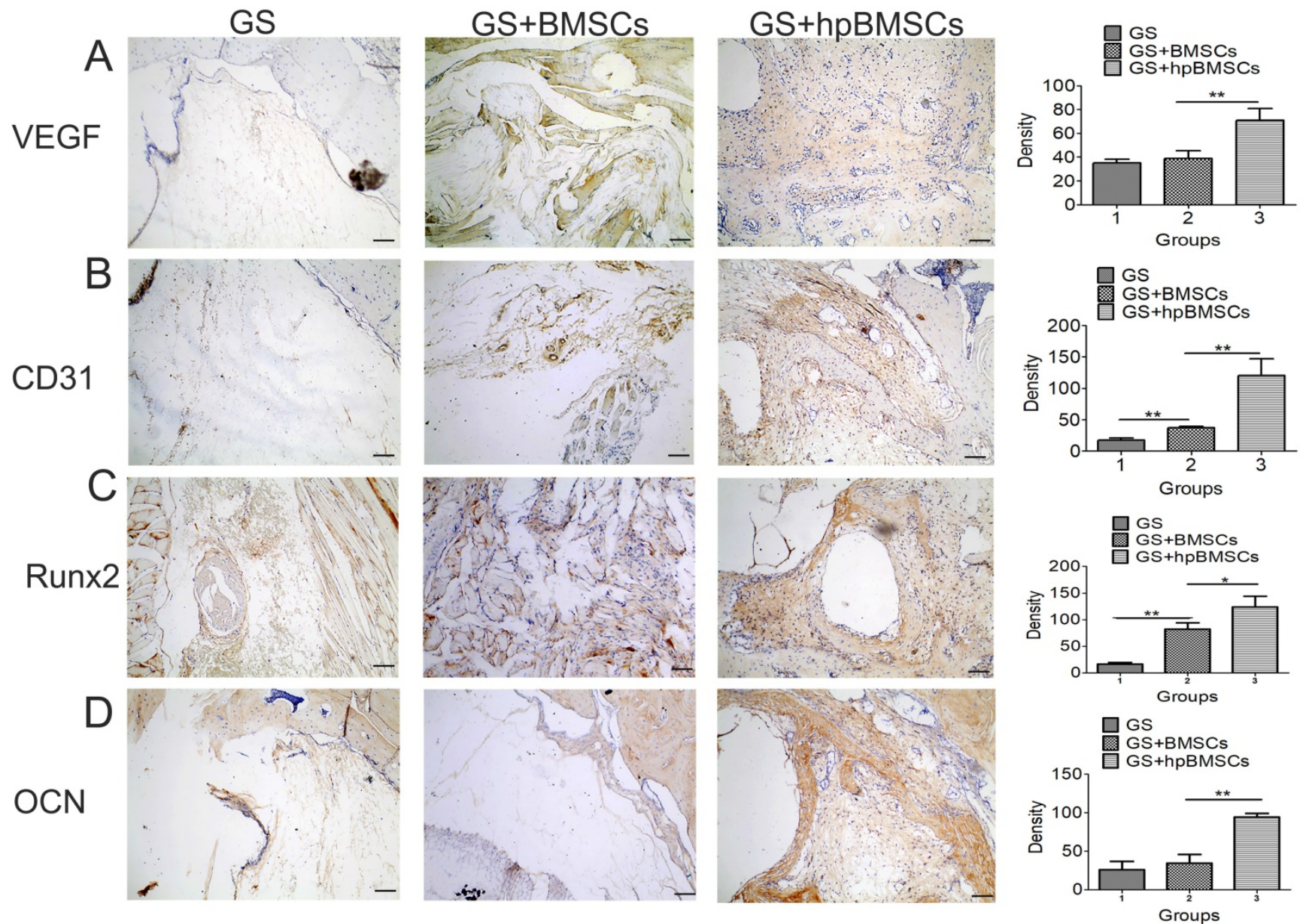

Figure 8. Immunohistochemical analysis of VEGF, CD31, Runx2 and OCN in the mandible defect area of aged rats 12 weeks after implantation. (A, B) Blood vessels were defined with the positive VEGF and CD31 stain. Immunohistochemistry for VEGF and CD31 showed there were more newly formed blood vessels in hypoxia preconditioned BMSCs Group than those in other groups. (C, D) Bone regeneration activity was indicated by the positive Run $\times 2$ and $O C N$ stain. There were no obvious positive staining for Run $\times 2$ and $O C N$ in GS group and GS+BMSCs group. While positive staining for Run $\times 2$ and OCN were apparent in GS+hp BMSCs group. (Scale bars $=50 \mu \mathrm{m}$. hpBMSCs, hypoxia preconditioned BMSCs.) 


\section{Discussion}

BMSCs are characterized as undifferentiated cells and possess a mesodermal differentiation potential, which participates in and plays a key role in the healing of bone injuries and bone regeneration. In general, the younger one creature is, the more BMSCs there are, and vice versa. Also, the biological characters of endogenous BMSCs have age-related changes, namely the osteo-/adipogenic transdifferentiation phenomenon, the osteogenic tendency decreases with aging while the adipogenic tendency increases [24-26]. In previous studies of osteogenic differentiation of aged BMSCs, notch signaling pathway was up regulated in aged individuals, and osteoblast differentiation was suppressed to maintain the number of bone marrow mesenchymal progenitors [27-28]. This phenomenon severely decreases the ability of bone regeneration of BMSCs and thus affected the speed and quality of bone defects regeneration, which disturbed the surgical treatment and prognosis. Our previous study demonstrated that using $\gamma$-secretase inhibitor can revert the notch signaling related attenuation of osteogenic differentiation in aged bone marrow mesenchymal stem cells [29]. Based on the above cognition, increasing the BMSCs number in bone defects area of the aged individuals is important.

Bone defect is often linked to ischemia and hypoxia. It is important to detect the reaction of BMSCs to hypoxia and trophic factor deprivation condition. Using CCK-8, we found that the proliferation capacity of BMSCs cultured under $1 \% \mathrm{O}_{2}$ condition decreased to a minimum in $48 \mathrm{~h}$ and then increased. And the CFU-F assay showed that BMSCs under $1 \% \mathrm{O}_{2}$ condition formed more colonies. And these results indicate that the proliferation capacity of BMSCs cultured under $1 \% \mathrm{O}_{2}$ condition was inhibited in $48 \mathrm{~h}$, however, concerning a longer period was stimulated. The inhibition period may be a process that BMSCs adapt to hypoxia and the period continued for about $48 \mathrm{~h}$. It was reported that about $90 \%$ of transplanted MSCs died on the first day, and no more than $1 \%$ survived 4 days after transplantation $[30,31]$. HIF-1a up-regulation in cancer cells and neurons participates in cell survival and cell apoptosis under hypoxic conditions or trophic factor deprivation. In this study, BMSCs were hypoxically preconditioned in three ways. The HIF-1a protein expression, but not gene expression, was up-regulated and thus inhibits apoptosis induced by hypoxia. Live \& Dead Viability Assay showed that the survival rate of hypoxia preconditioned BMSCs was higher than that of regular BMSCs in $1 \% \mathrm{O}_{2}$ and serum-free medium condition. We demonstrated that hypoxia preconditioning inhibits BMSCs death induced by severe condition. Therefore, hypoxia preconditioning may enhance BMSCs viability in the bone defect area after transplantation and keep the number of BMSCs at a high level in bone defects thereby improve the bone healing capacity of BMSCs.

Previous studies demonstrated that HIF-1a up-regulation could directly enhance the osteogenic differentiation potential of BMSCs. A number of studies have investigated that using lentivirus mediated delivery of wild-type or constitutively active HIF-1a to enhance the expression level of HIF-1a [32]. However, the delivery of wild-type or constitutively active HIF-1a will lead to HIF-1a up-regulation in BMSCs forever, which may result in neoplasia. Some scholars have demonstrated that DMOG enhances survival rate and angiogenesis of cells in vitro and after transplantation into the ischemic condition [33]. In this study, we hypoxia preconditioned BMSCs with DMOG under $1 \% \mathrm{O}_{2}$ condition enhancing the survival rates during precondition process. On the other hand, DMOG promotes BMSCs osteogenic differentiation [34-35]. In this study we investigated a therapeutic strategy based on hypoxia preconditioned BMSCs with DMOG under $1 \% \mathrm{O}_{2}$ for healing critical-size mandible defect in aged individuals.

Angiogenesis is a complex process, which needs the involvement of multiple angiogenic factors and Hif-1a participates in the regulation of angiogenesis. Hif- $1 \alpha$ is stabilized and accumulated after hypoxia precondition, and then binds to HIF-1 $\beta$ forming HIF-1 complex. HIF-1 complex then stimulates the expression of VEGF [36]. VEGF is a potent mitogen for endothelial cells and plays a central role in angiogenesis and neovascularization [37]. In this study, tube formation test showed hypoxia preconditioned BMSCs formed more tubes and the total tube length was significantly longer than that formed by regular BMSCs. Western blotting demonstrated that the expression level of VEGF in BMSCs were also up regulated after hypoxia preconditioning, and the $1 \% \mathrm{O}_{2}+0.5 \mathrm{mM}$ DMOG preconditioned group was the highest. The qRT-PCR result showed that VEGF mRNA expression of hypoxia preconditioned BMSCs was significantly higher compared that of in regular BMSCs. Using ELISA Kit to detect the secretion of VEGF, we found that hypoxia preconditioning for $48 \mathrm{~h}$ led to an obvious elevation. And the super numerary release of VEGF is thought to be crucial for angiogenesis in bone regeneration [38]. The processes of angiogenesis and osteogenesis are coupled during bone regeneration [39]. Blood vessels not only transport oxygen and nutrients but also play an important role in bone 
formation and remodeling by mediating the interaction between osteoblasts, osteocytes, osteoclasts, and vascular cells [40]. These data confirmed that hypoxia preconditioned BMSCs had a better angiogenic capacity in vitro.

Bone regeneration capacity of BMSCs after they were hypoxia preconditioned was evaluated in vitro. Alkaline phosphatase activity assay and Alizarin red staining were taken under $1 \% \mathrm{O}_{2}$ condition. And the results reveal that the capacity of hypoxia preconditioned BMSCs for osteogenic differentiation was significantly enhanced as compared with that of in regular BMSCs. These data confirmed that hypoxia preconditioned BMSCs had a better osteogenic capacity in vitro. To detect the osteogenic capacity of hypoxia preconditioned BMSCs in aged rats, a critical-sized defect on the mandible of aged rats was made, hypoxia preconditioned BMSCs were implanted into critical-sized mandible defects in aged rats to explore their capability of promoting bone healing in vivo. The micro-CT and immunohistochemistry results showed that hypoxia preconditioning could improve the capacity of BMSCs for bone regeneration in aged rats in vivo. The H\&E, Mallory's trichrome staining and Immunohistochemistry showed that most of the newly formed tissue in GS group was muscular tissue, while the in GS+hypoxia reconditioned BMSCs group was fibrous tissues and bone, and we could confirm that the repair of the bone defect in aged rats is difficult and slow. The participation of hypoxia preconditioned BMSCs significantly improved the bone repair quality.

Endogenous and environmental inflammatory response can contribute to cell death and it is believed that the decrease in bone formation serves as a major cause of the age-related bone loss [41, 42]. Moreover, there are fewer osteoblasts in aged bones than in young bones $[43,44]$. It has been well known that BMSCs are the precursor cells for many cell types, and among them is osteoblast [45]. And the regulation of notch and other signaling path-ways in aged individuals suppresses the differentiation of BMSCs into osteoblasts [46]. Thus, improving grafted cell survival rate after transplantation to increase the number of survived BMSCs in bone defect area is critical for enhancing the efficacy and efficiency of stem cell therapy. In this study, the combination of hypoxia and DMOG preconditioning significantly increased the survival rate in bone defect site of transplanted BMSCs, and may have great potential in regenerative cell therapy for bone defects in aged individuals.

In conclusion, this study demonstrated that hypoxia preconditioning BMSCs with the combination of $1 \% \quad \mathrm{O}_{2}$ and DMOG treatment increased the survival rate of the transplanted BMSCs in the severe condition by stabilizing the expression of HIF-1a in cells. And increase the BMSCs number in the aged bone marrow. Hypoxia preconditioning in vitro could improve the angiogenesis and osteogenesis activity of BMSCs under hypoxia in vivo. The results provide an evidence for the further application of BMSCs transplantation promoting bone regeneration in aged population.

\section{Abbreviations}

BMSCs: bone marrow mesenchymal stem cells; HIF-1a: Hypoxia inducible factor-1a; DMOG: Dimethyloxaloylglycine; MEM: minimum essential medium; VEGF: vascular endothelial growth factor; CCK-8: Cell Counting Kit-8; CFU-F: Colony-Forming Unit-Fibroblast; ALP: Alkaline phosphatase; ARS: Alizarin Red S; GS: gelatin sponge; hpBMSCs: hypoxia preconditioned BMSCs.

\section{Acknowledgments}

This study was supported by the Natural Science Foundation of China (81571366, 81270421) and Sichuan Province Science and Technology Innovation Team Program (2017TD0016).

\section{Competing Interests}

The authors have declared that no competing interest exists.

\section{References}

1. Abdallah BM, Kassem M. New factors controlling the balance between osteoblastogenesis and adipogenesis. Bone. 2012; 50: 540-5

2. Gao B, Yang L, Luo Z-J. Transdifferentiation between bone and fat on bone metabolism. Int J Clin Exp Pathol. 2014; 7: 1834-41

3. PACHÓN-PEÑA G, Yu G, Tucker A, WU X, Venrdrell J, Bunnell BA, et al. Stromal Stem Cells From Adipose Tissue and Bone Marrow of Age-Matched Female Donors Display Distinct Immunophenotypic Profiles. Journal of cellular physiology. 2011; 226(3): 843-51.

4. Díaz D, Recio JS, Baltanás FC, Gómez C, Weruaga E, Alonso JR, et al. Long-lasting changes in the anatomy of the olfactory bulb after ionizing irradiation and bone marrow transplantation. In Neuroscience; 2011; 173: 190-205.

5. Lendahl U, Lee KL, Yang H, Poellinger L. Generating specificity and diversity in the transcriptional response to hypoxia. NATURE REVIEWs Genetics; 2009; 10:821-32.

6. Kallio PJ, Pongratz I, Gradin K, McGuire J, Poellinger L. Activation of hypoxia-inducible factor 1a: posttranscriptional regulation and conformational change by recruitment of the Arnt transcription factor. Proc Natl Acad Sci; 1997; 94: 5667-72.

7. Salceda S, Caro J. Hypoxia-inducible factor $1 a(\mathrm{HIF}-1 \mathrm{a})$ protein is rapidly degraded by the ubiquitin-proteasome system under normoxic conditions. Its stabilization by hypoxia depends on redox-induced changes. J. Biol. Chem; 1997; 272: 22642-647.

8. Pittenger MF, Mackay AM, Beck SC. Multilineage potential of adult human mesenchymal stem cells. Science. 1999; 284: 143-7.

9. Prockop DJ. Marrow stromal cells as stem cells for nonhematopoietic tissues. Science. 1997; 276: 71-4.

10. Fehrer C, Brunauer R, Laschober G. Reduced oxygen tension attenuates differentiation capacity of human mesenchymal stemcells and prolongs their life span. Aging Cell. 2007; 6: 745-57.

11. Lennon DP, Edmison JM, Caplan AI. Cultivation of rat marrow-derived mesenchymal stem cells in reduced oxygen tension: Effects on in vitro and in vivo osteochondrogenesis. J Cell Physiol. 2001; 187: 345-55.

12. Semenza GL. Regulation of mammalian $\mathrm{O}_{2}$ homeostasis by hypoxia-inducible factor 1. Annu. Rev.Cell Dev. Biol. 2014; 15: 551-78. 
13. Liu L, Simon MC. Regulation of transcription andtranslation by hypoxia. Cancer Biol. Ther. 2004; 3: 492-7.

14. $\mathrm{Hu} \mathrm{X}$. Transplantation of hypoxia-preconditioned mesenchymal stem cellsimproves infarcted heart function via enhanced survival of implanted cells and angiogenesis. J. Thorac. Cardiovasc. Surg. 2008; 135: 799-808.

15. Maniatopoulos C, Sodek J, Melcher AH. Bone formation in vitro bystromal cells obtained from bone marrow of young adult rats. Cell Tissue Res. 1988; 254: 317-30.

16. Baksh D, Tuan RS. Canonical and noncanonical Wnts differentially affect the development potential of primary isolate of human bone marrow mesenchymal stem cells. J CellPhysiol. 2007; 212: 817-26.

17. Boyette LB, Creasey OA, Guzik L, Lozito T, Tuan RS. Human Bone Marrow-Derived Mesenchymal Stem Cells Display Enhanced Clonogenicity but Impaired Differentiation with Hypoxic Preconditioning. Stem Cells Translational Medicine. 2014; 3(2): 241-54.

18. Baustian C, Hanley S, Ceredig R. Isolation, selection and culture methods to enhance clonogenicity of mouse bone marrow derived mesenchymal stromal cell precursors. Stem Cell Research \& Therapy. 2015; 6(1): 151-9.

19. Misra MK, Sarwat M, Bhakuni P, Tuteja R, Tuteja N. Oxidative stress and ischemic myocardial syndromes. Med Sci Monit. 2009; 15: 209-19.

20. Liu XB, Wang JA, Ji XY, Yu SP, Wei L. Preconditioning of Bone Marrow Mesenchymal Stem Cells by Prolyl Hydroxylase Inhibition Enhances Cell Survival and Angiogenesis in vitro and after Transplantation into the Ischemic Heart of Rats. Stem Cell Research \& Therapy. 2014; 5(5): 111-6.

21. Zou DH, Zhang ZY, YE DX, Tang AF, Deng LF, Han W, et al. Repair of Critical-Sized Rat Calvarial Defects Using Genetically Engineered Bone Marrow-Derived Mesenchymal Stem Cells Overexpressing Hypoxia-Inducible Factor-1a. STEM CELLS. 2011; 29: 1380-90.

22. Rasubala L, Yoshikawa H, Islam AAS, Nagata k,Iijima T, Ohishi M. Comparison of the healing process in plated and non-plated fractures of the mandible in rats. British Journal of Oral and Maxillofacial Surgery. 2004; 42: $315-22$.

23. Fan J, Park H, Lee MK, Bezouglaia O, Fartash A, Kim J. et al. Adipose-Derived Stem Cells and BMP-2 Delivery in Chitosan-Based 3D Constructs to Enhance Bone Regeneration in a Rat Mandibular Defect Model. Tissue Engineering. Part A. 2013; 20: 2169-79.

24. Gao B, Yang L, Luo ZJ. Transdifferentiation between bone and fat on bone metabolism. Int J Clin Exp Pathol. 2014; 7: 1834-40.

25. Ullah $M$, Sittinger $M$, Ringe J. Transdifferentiation of adipogenically differentiated cells into osteogenically or chondrogenically differentiated cells: phenotype switching via dedifferentiation. Int J Biochem Cell Biol. 2014; 46: 124-37.

26. Liu W, Zhou L, Zhou C, Zhang S, Jing J, Xie L, et al. GDF11 decreases bone mass by stimulating osteoclastogenesis and inhibiting osteoblast differentiation. Nat Commun. 2016; 22(7): 12794.

27. Liu J, Sato C, Cerletti M, Wagers A. Notch signaling in the regulation of stem cell self-renewal and differentiation. Curr Top Dev Biol. 2010; 92: 367-409.

28. Fukada SI, Ma Y, Uezumi A. Adult stem cell and mesenchymal progenitor theories of aging. Front Cell Dev Biol. 2014; 2:10.

29. Tang Z, Wei J, Yu Y, Zhang J, Liu L, Tang W, et al. Y-Secretase inhibitor reverts the Notch signaling attenuation of osteogenic differentiation in aged bone marrow mesenchymal stem cells. Cell Biol Int.2016; 40: 439-47.

30. Zou DH, Zhang ZY, YE DX, Tang AF, Deng LF, Han W, et al. Repair of Critical-Sized Rat Calvarial Defects Using Genetically Engineered Bone Marrow-Derived Mesenchymal Stem Cells Overexpressing Hypoxia-Inducible Factor-1a. STEM CELLS. 2011; 29: 1380-90.

31. Lee TJ, Bhang SH, Yang HS, La WG, Yoo HH, Shin JY, et al. Enhancement of long-term angiogenic efficacy of adipose stem cells by delivery of FGF2. Microvasc Res. 2012; 1: 1-8.

32. Assmus B, Honold J, Schachinger V, Britten MB, Fischer RU, Lehmann R, et al. Transcoronary transplantation of progenitor cells after myocardial infarction. N Engl J Med. 2012; 12: 1222-32.

33. Liu XB, Wang JA, Ji XY, Yu SP, Wei L. Preconditioning of Bone Marrow Mesenchymal Stem Cells by Prolyl Hydroxylase Inhibition Enhances Cell Survival and Angiogenesis in vitro and after Transplantation into the Ischemic Heart of Rats. Stem Cell Research \& Therapy. 2014; 5(5): 111-6..

34. Zhang L, Jiang G, Zhao X, Gong Y. Dimethyloxalylglycine Promotes Bone Marrow Mesenchymal Stem Cell Osteogenesis via Rho/ROCK Signaling. Cell Physiol Biochem. 2016; 39: 1391-1403.

35. Peng J, Lai ZG, Fang ZL, Xing S, Hui K, Hao C, et al. Dimethyloxalylglycine Prevents Bone Loss in Ovariectomized C57BL/6J Mice through Enhanced Angiogenesis and Osteogenesis. PLoS ONE. 2014; 9(11): e112744.

36. Woo KM, Jung HM, Oh JH, Rahman SU, Kim SM, Baek JH, et al. Synergistic effects of dimethyloxalylglycine and butyrate incorporated into a-calcium sulfate on bone regeneration. Biomaterials. 2015; 39: 1-14.

37. Amelio I, Inoue S, Markert EK, Levine AJ, Knight RA, Mak TW, et al. TAp73 opposes tumor angiogenesis by promoting hypoxia-inducible factor $1 \mathrm{a}$ degradation. PNAS. 2015; 112(1): 226-31.

38. Bitar MS, Al-Mulla F. Upregulation of CREM/ICER suppresses wound endothelial CRE-HIF-1a-VEGF-dependent signaling and impairs angiogenesis in type 2 diabetes. Disease Models \& Mechanisms. 2015; 8(1): 65-80.

39. Ding $\mathrm{H}$, Chen $\mathrm{S}$, Song $\mathrm{W}$, Gao $\mathrm{Y}$, Guan J, Wang $\mathrm{Y}$, et al Dimethyloxaloylglycine Improves Angiogenic Activity of Bone Marrow Stromal Cells in the Tissue-Engineered Bone. International Journal of Biological Sciences. 2014; 10(7): 746-56.
40. Yang KC, Wang CC, Wu CC. Acute and subacute oral toxicity tests of sintered dicalcium pyrophosphate onovariectomized rats for osteoporosis treatment. Biomed Eng-App Bas C. 2010; 22: 169-76.

41. Riddle RC, Khatri R, Schipani E, Clemens TL. Role of hypoxia-inducible factor-1a in angiogenic-osteogenic coupling. Journal of molecular medicine (Berlin, Germany). 2009; 87(6): 583-590.

42. Guan M, Yao W, Liu R, Lam KS, Nolta J, Jia J, et al. Directing mesenchymal stem cells to bone to augment bone formation and increase bone mass. Nat Med. 2012; 18: 456-62.

43. Rosen CJ, Bouxsein ML. Mechanisms of disease: is osteoporosis the obesity of bone? Nat Rev Rheumatol. 2006; 2: 35-43.

44. Duque G, Rivas D, Li W, Li A, Henderson JE, Ferland G, et al. Age-related bone loss in the LOU/c rat model of healthy ageing. Exp Gerontol. 2009; 44: 183-9.

45. Shuai Y, Liao L, Su X, Yu Y, Shao B, Jing H, et al. Melatonin Treatment Improves Mesenchymal Stem Cells Therapy by Preserving Stemness during Long-term In vitro Expansion. Theranostics. 2016; 6: 1899.

46. Hilton MJ, Tu X, Wu X, Bai S, Zhao H, Kobayashi T, et al. Notch signaling maintains bone marrow mesenchymal progenitors by suppressing osteoblast differentiation. Nat Med. 2008; 14: 306-14. 